\title{
Leveraging Semantic Technologies towards Social Ambient Intelligence
}

\author{
Adrien JOLY ${ }^{1,2}$, Pierre MARET $^{2}$ and Fabien BATAILLE ${ }^{1}$ \\ ${ }^{1}$ Alcatel-Lucent Bell Labs \\ ${ }^{2}$ LIRIS / INSA Lyon \\ Centre de Villarceaux \\ Route de Villejust \\ 91620 Nozay, France \\ adrien.joly@alcatel-lucent.fr \\ fabien.bataille@alcatel-lucent.fr \\ Bâtiment Blaise Pascal \\ 20, avenue Albert Einstein \\ 69621 Villeurbanne Cedex, France \\ adrien.joly@liris.cnrs.fr \\ pierre.maret@insa-lyon.fr
}

\begin{abstract}
At a time when the amount of information grows exponentially on the Internet, when most people can be connected at all times with powerful personal devices, we need to enhance, adapt and simplify access to information and communication with other people. The vision of ambient intelligence which is a relevant solution, brings many challenges in the domain of contextawareness, adaptive human-system interaction, privacy enforcement and social communications. We believe that ontologies and other semantic technologies can help to meet most of these challenges in a unified manner, as they are a bridge between meaningful (but fuzzy by nature) human knowledge and digital information systems. In this chapter, we review several utilizations of semantic technologies for context management, adaptive human-system interaction, privacy enforcement and social communications in the scope of ubiquitous computing. Based on identified benefits and lacks, we propose several research leads towards the realization of a unified ubiquitous system based on the social web and semantic technologies.
\end{abstract}

\section{Introduction}

At a time when the amount of information grows exponentially on the Internet, when most people can be connected at all times with powerful personal devices, users suffer from the growing complexity of the information society. Our usage of technology is moving towards the vision of "Ambient Intelligence", derived from the vision of "Ubiquitous computing" in which "the most profound technologies are those that disappear" (Weiser, 1991) and thus access to information is not longer limited to personal computers and the web browsing paradigm. This vision brings many technological and psychological challenges (Streitz \& Nixon, 2005) that are considered in several research domains, including:

- Context-awareness: how to take one's context into account to improve his communication?

- Multimodality: how to span user interfaces from a terminal into separate modal interfaces ? (e.g. various screens, input controllers, microphones, phones...)

- Social networking: how to enhance and leverage social communication ?

- Privacy \& Trust: how to ease one's life without delegating human control to machines?

There is one transversal question yet to answer: is there a unified approach that could answer these challenges in a global way that makes sense ? Actually, there exists a common approach that is considered in all these research domains, and in most corresponding works it has been shown as very promising. This approach is the utilization of semantic technologies.

In this chapter, we propose a review of research works relying on semantic technologies towards ambient intelligence and its social applications. The intention here is to identify the key 
technologies, approaches and issues that may be blended in order to build an optimal platform for a widescaled ubiquitous system. After defining the foundational terms of this chapter in the Background section, we will review several research works to identify their key technologies, approaches and issues in the State-of-the-Art section, then we will propose several research leads towards an optimal ubiquitous framework based on semantic technologies in the Future Trends section, to finally conclude this chapter.

\section{Background}

In this section, we propose and discuss the underlying definitions for the foundations of this chapter: ubiquitous computing, context-awareness and semantic technologies.

\section{Ubiquitous Computing, Ambient Intelligence and Context-awareness}

The phrase "ubiquitous computing" was proposed by Mark Weiser while working for the Xerox Palo Alto Research Center (PARC), to qualify a possible evolution of computers. "The Computer for the $21^{\text {st }}$ century" (Weiser, 1991) has become a foundational paper for following works in this domain, introducing his vision in which "ubiquitous computers" are simple communicative devices and appliances that are suited for a particular task and know where they are while fading into the background. For example, paper sheets could be replaced with flexible screens, bringing any information of the web as an independent element of your real desktop, an element that you can stack into piles, stick on a wall, lend to a colleague or take with you for lunch.

As depicted on Figure 1, we have already reached the generation of ubiquitous computers, as powerful and communicative computers are spread in many devices like watches, mobile phones, portable media players and game consoles, PDAs, ticket machines, bike renting beacons and kids toys. Even though we have not reached Mark Weiser's vision of interoperable and shared ubiquitous computers, a significant research effort is done towards the vision of "Ambient intelligence", an evolution of "ubiquitous computing" in which networked devices can also be integrated in the environment (and thus not expecting any user intervention), can sense the environmental, personal and social situation to adapt the experience, and can anticipate forthcoming situations or actions.

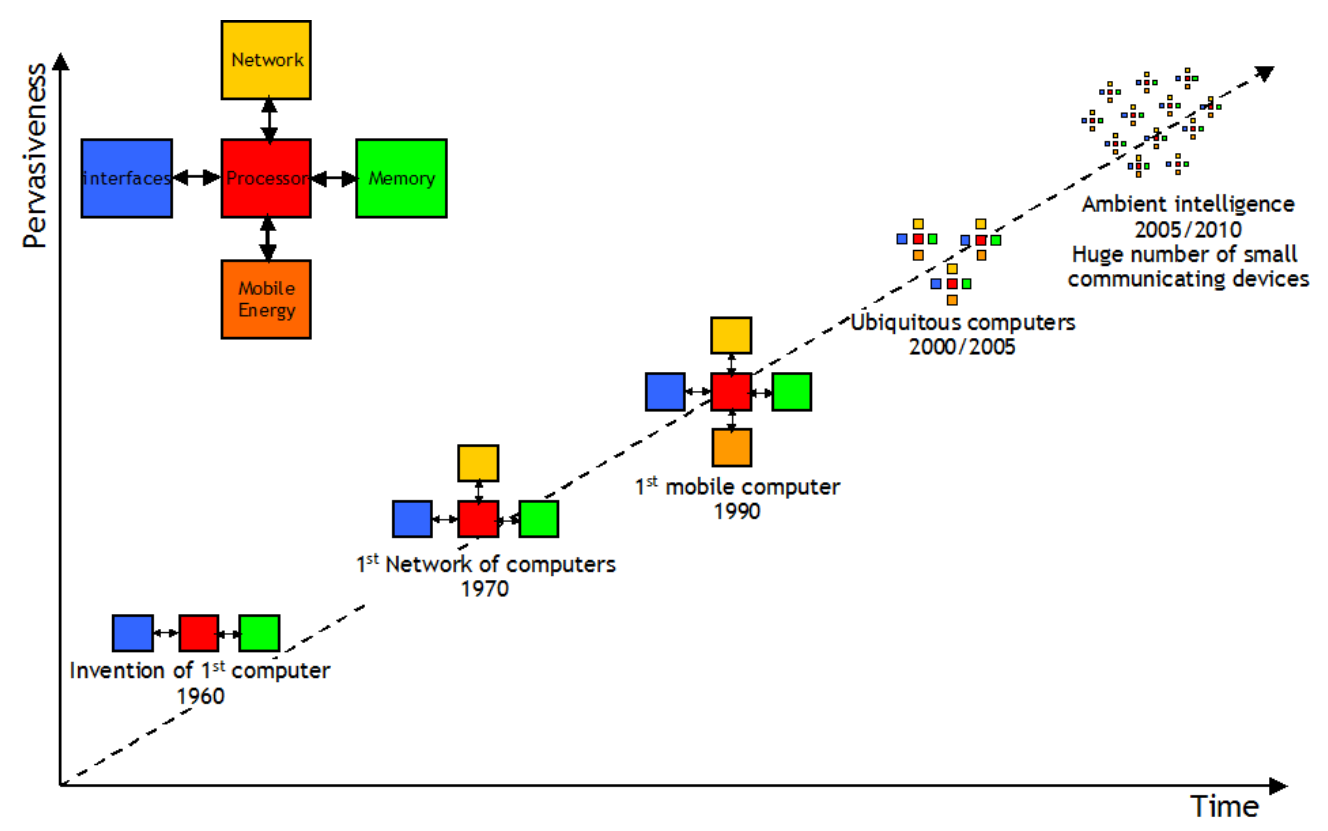

Figure 1: The evolution of computing, from "Nano computing \& Ambient intelligence" (Waldner, 2007) 
Context-awareness is a key research domain towards the vision of Ambient Intelligence. It consists of acquiring low-level context data (e.g. from sensors), inferring high-level knowledge from this data, and predict context changes in order to improve the user experience transparently. As depicted on Figure 2, the first level of context contains current raw sensor data like GPS coordinates, IP address, surrounding Bluetooth MAC addresses or temperature. By combining and inferring on this knowledge, a meaningful position or activity like "in a meeting" or "watching TV" can be deduced on the second level. Then, after having learned the habits of the user, the third level can predict what action is probably going to happen next or whether exceptional cases have occurred (e.g. the user is going to arrive late at work because he has not left home yet) in order to undertake relevant actions (e.g. inform the colleagues that the meeting is delayed).

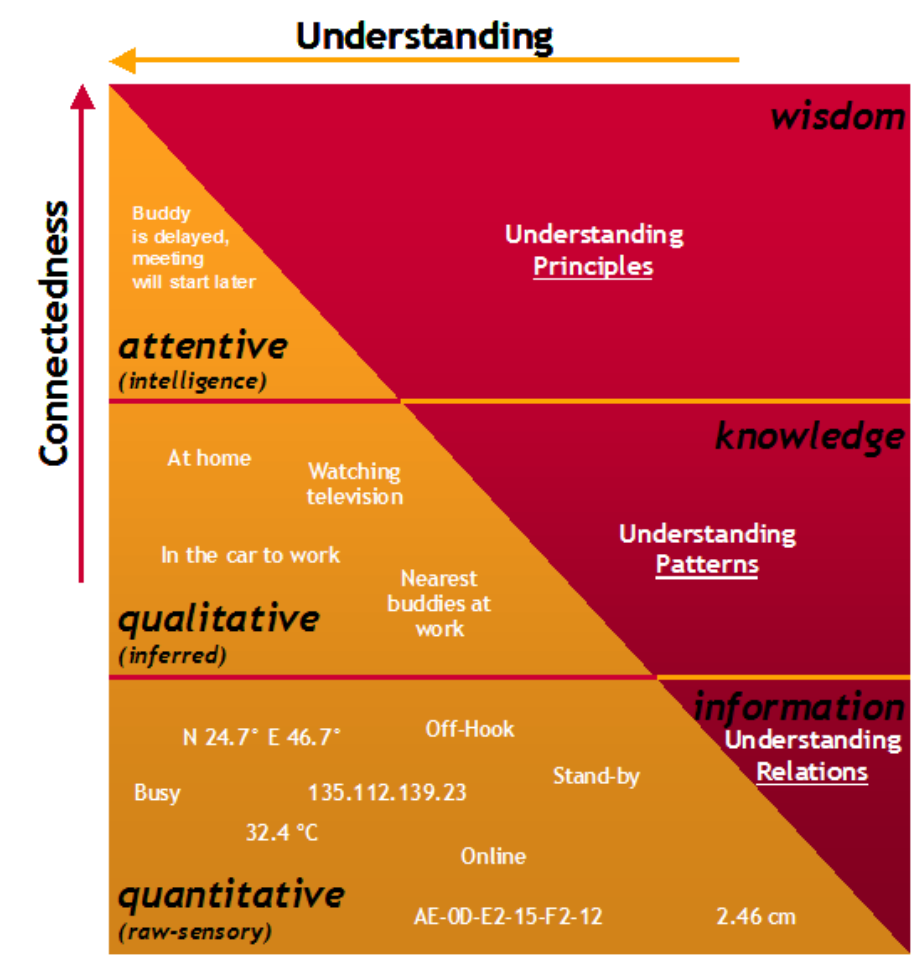

Figure 2: Levels of context (Bell Labs)

\section{Semantic technologies: ontologies, knowledge representation and reasoning}

In their study, (Strassner, O'Sullivan, \& Lewis, 2007) define ontologies as « a formal, explicit specification of a shared, machine-readable vocabulary and meanings, in the form of various entities and relationships between them, to describe knowledge about the contents of one or more related subject domains throughout the life cycle of its existence ». Semantic technologies, including ontologies and semantic description languages, are quite similar to human thinking and memorization: they allow the definition of concepts and instances (of these concepts) that are related with each other using semantically qualified links, the extraction of knowledge from a base and reasoning on this knowledge to deduce inferred knowledge. Applying such an approach to information technologies enable machines to understand the actual meaning of data which is formulated using a distributed and evolving vocabulary. That way, ontologies fill the gap between ambiguous/fuzzy human thinking (e.g. in natural languages, a word can have different meanings) and formalized digital data (i.e. stored using specific formats and interpreted by specific applications for a specific purpose). As an example, most popular search engines of the Internet are keyword-based and thus base their queries on the occurrence of given words in the textual content of pages. The drawback of this approach is that words can have very different meanings (interpretations), and also a concept can be formulated with many different words; therefore if I 
would like to eat an apple and search for an " apple store », the search engine will probably propose a store vending computers and MP3 players! On the other hand, a semantic search engine would ask you if you are looking for fruits or electronic devices to return relevant results. If we expect a system to bring relevant information existing on any domain of interest, it must reason on meaningful knowledge, and semantic technologies are identified as the most promising candidate to enable this.

One of the benefits of using semantic languages is to allow progressive/incremental modeling of a system, reflecting the natural progression of conceptual understanding of domains. Ontologies can ease the communication between heterogeneous networked entities (i.e. using different languages/protocols) by matching similar portions of the semantic graph of the sender's knowledge with the recipient's knowledge.

We will now investigate on how semantic technologies have been utilized in ubiquitous contextaware systems and identify future trends on this domain.

\section{State-of-the-Art}

Previous studies (Strang \& Linnhoff-Popien, 2004; Baldauf, Dustdar, \& Rosenberg, 2007; O. Lassila \& Khushraj, 2005) have identified ontologies as the most promising enabler for ubiquitous context-aware systems because they are heterogeneous and extensible by nature, and semantic technology enables « future-proof » interoperability. In this section, we will study the utilization of semantic technologies in four aspects of ambient intelligence: context management, human-system interactions, privacy enforcement, and social communications.

\section{Semantic context management}

According to (Dey, 2001), "a system is context-aware if it uses context to provide relevant information and/or services to the user, where relevancy depends on the user's task". By context, Dey means "any information that can be used to characterize the situation of an entity. An entity is a person, place, or object that is considered relevant to the interaction between a user and an application, including the user and applications themselves".

(Gu, Wang, Pung, \& Zhang, 2004) gave an introduction to context-awareness by proposing the following requirements: «An appropriate infrastructure for context-aware systems should provide support for most of the tasks involved in dealing with contexts - acquiring context from various sources such as physical sensors, databases and agents; performing context interpretation; carrying out dissemination of context to interested parties in a distributed and timely fashion; and providing programming models for constructing of context-aware services. »

The use of ontologies to store and manipulate context impacts other aspects of the underlying system: context knowledge exchange, learning, user interactions, security and applications. In this section we will review several semantic-based approaches for context management platforms and identify the most successful approaches and current lacks.

\section{Review of major context-aware platforms}

One of the first semantic context modeling approaches was the Aspect-Scale-Context (ASC) model proposed by (Strang, Linnhoff-Popien, \& Frank, 2003). Compared to non-semantic models, ASC enabled contextual interoperability during service discovery and execution in a distributed system. Indeed, this model consists in three concepts:

- Aspects are measurable properties of an entity (e.g. the current temperature of a room)

- Scales are metrics used to express the measure of these properties (e.g. Celsius temperature)

- Context qualifies the measure itself by defining the sensor, the timestamp and quality data 
Contexts can be converted from a scale to another using Operations, also described semantically, and can be mapped to an implemented service. This model has been implemented as the CoOL Context Ontology Language. The CoOL core ontology can be formulated in OWL-DL (Dean \& Schreiber, 2004) and F-Logic (object-oriented). The CoOL integration is an extension of the core to inter-operate with web services. OntoBroker (Decker, Erdmann, Fensel, \& Studer, 1999) was chosen for semantic inference and reasoning, supporting F-Logic as knowledge representation and query language.

With EasyMeeting, (Chen et al., 2004) proposed a pragmatic application to demonstrate the benefits of their semantic context-aware system called CoBrA, for Context Broker Architecture. This application assists a speaker and its audience in a meeting situation by welcoming them in the room, dimming the lights, and displaying the presentation slides, either by vocal commands or automatically. The underlying prototype that they developed is a multi-agent system based on JADE (Java Agent DEvelopment Framework) [http://sharon.cselt.it/projects/jade/] in which a broker maintains a shared context model for all computing entities by acquiring context knowledge from various sensors and by reasoning on this knowledge to make decisions, as depicted on Figure 5. In the EasyMeeting application, this broker can deduce the list of expected participants and their role in the meeting by accessing their schedule and can sense their actual presence when the bluetoothenabled mobile phone declared in their profile is detected in the room. That way, the system can notify the speaker about their presence and decide to dim the lights and turn off the music when he arrives. These decisions are made possible by reasoning on the context knowledge using rules defined by the EasyMeeting application. The context knowledge is represented as RDF triples relying on the COBRA-ONT OWL ontology that includes vocabularies from the SOUPA ontology (Chen, Perich, Finin, \& A. Joshi, 2004) covering time, space, policy, social networks, actions, location context, documents, and events, as depicted on Figure 4. Inferencing on the OWL ontology is handled by JENA's API [http://jena.sourceforge.net] whereas the JESS rule-based engine [http://herzberg.ca.sandia.gov/] is used for domain-specific reasoning. The execution of rules (when results cannot be inferred from ontology axioms alone) uses the forward-chaining inference procedure of JESS to reason about contextual information. Note that, in this case, essential supporting facts must be extracted from RDF to JESS representation and the eventual results have to be injected in RDF to the knowledge base, which implies additional overhead in the process.
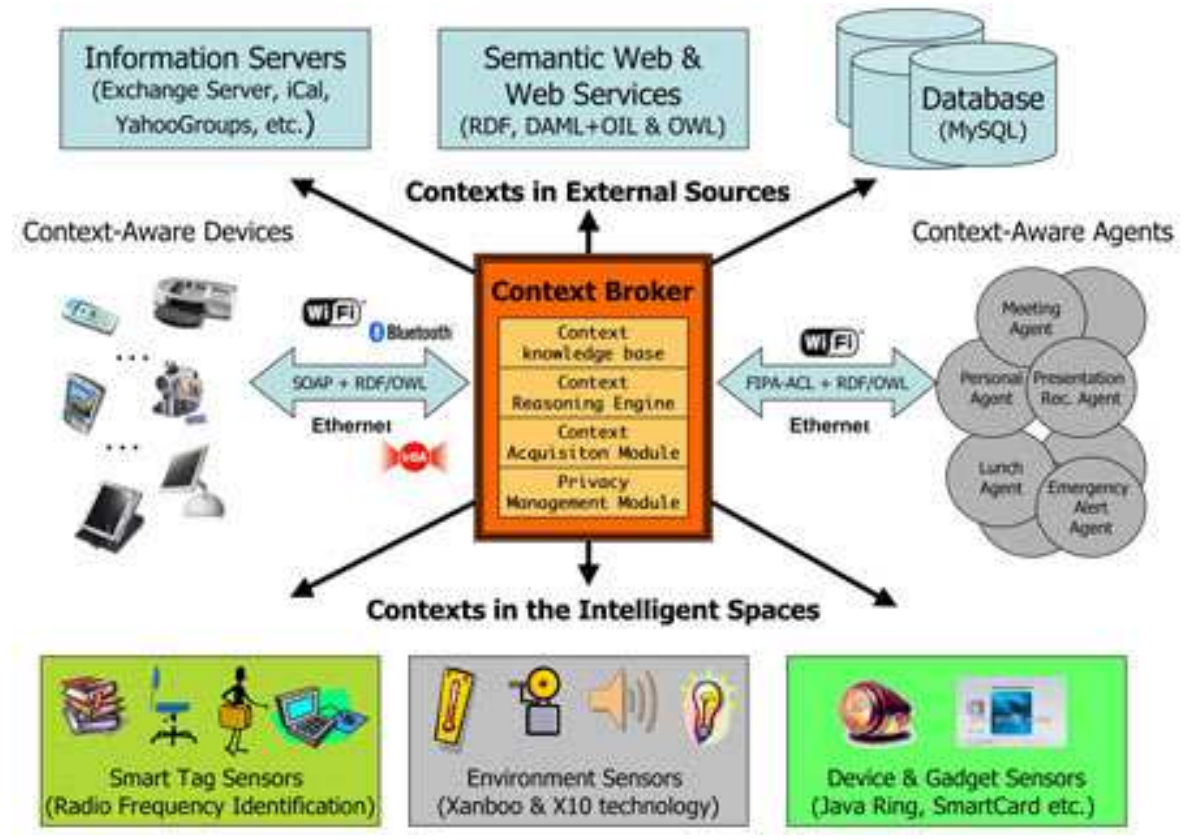

Figure 3: Overview of CoBrA

CoBrA's broker enforces privacy policies to define rules of behavior and restrict context 
communication. Enforcement of user-defined policies rely on the Rei role-based policy-reasoning engine (Kagal \& T. A. Joshi, 2003) which does description logic inference over OWL. CoBrA also implements a meta-policy reasoning mechanism so that users can override some aspects of a global policy to define specific constraints at their desired level of granularity. However, they do not provide a tool for the user to express his/her privacy policy.

The SOUPA ontology proposed by (Chen et al., 2004) and used in CoBrA was a collaborative effort to build a generic context ontology for ubiquitous systems. It has been maintained by the "Semantic Web in Ubiquitous Comp Special Interest Group" since 2003. The design of this ontology is driven by use cases and relies on FOAF, DAML-Time, OpenCyc (symbolic) + OpenGIS (geospatial) spatial ontology, COBRA-ONT, MoGATU BDI (human beliefs, desires and intentions) and Rei policy ontology (rights, prohibitions, obligations, dispensations). SOUPA defines its own vocabulary, but most classes and properties are mapped to foreign ontology terms using the standard OWL ontology mapping constructs (equivalentClass and equivalentProperty), which permit interoperability. In the core ontology in which both computational entities and human users can be modeled as agents, the following extensions are added: meeting \& schedule, document \& digital document, image capture, location (sensed location context of things).

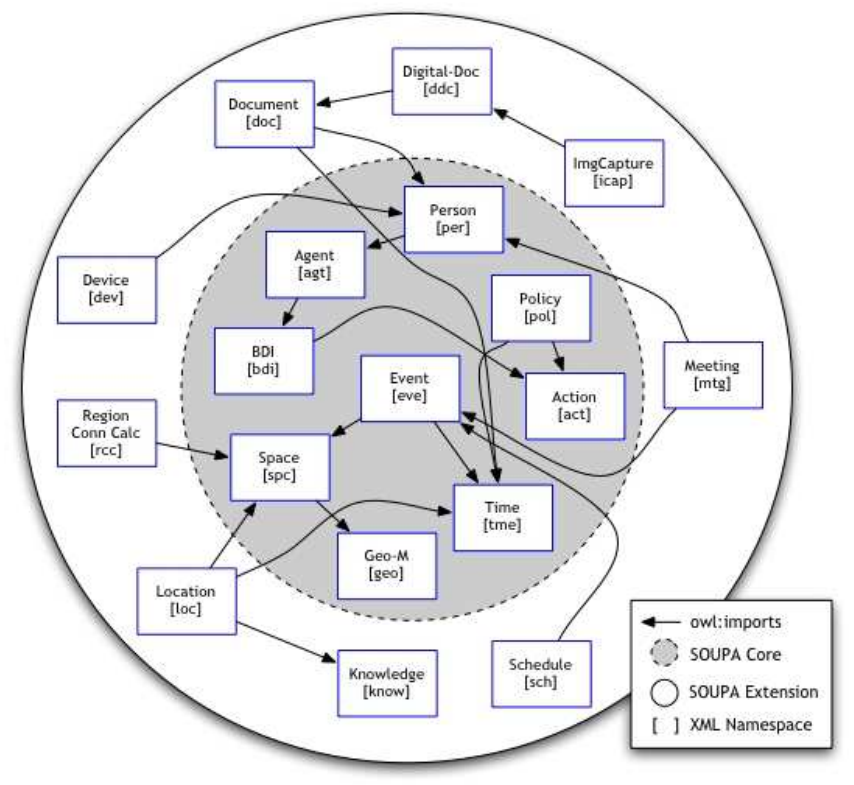

Figure 4: The SOUPA ontology

Like CoBrA, MOGATU (Perich, Avancha, Chakraborty, A. Joshi, \& Yesha, 2005) is a contextaware system based on the SOUPA ontology. However, this decentralized peer-to-peer multi-agent system implements several use cases covering automatic and adaptive itinerary computation based on real-time traffic knowledge, and commercial recommendation. In this approach, each device is a semi autonomous entity driven by the user's profile and context, relying on a contract-based transaction model. This entity is called InforMa and acts as a personal broker that handles exchanges with other peers. The user profile semantically defines his beliefs, desires and intentions, following the BDI model that is part of the SOUPA ontology. Beliefs are weighted facts depicting user knowledge and preferences such as his schedule and cuisine preferences, whereas desires express users' goals. Intentions are defined as a set of intended tasks that can be inferred from desires or explicitly provided. However no clues are given by the authors about how these beliefs and intentions are defined by the user or the system, which let us assume that this is still a manual process yet to be enriched with profiling mechanisms and a graphical user interface to edit the profile. Moreover, this work being apparently focused on trusted peer-to-peer exchange of information according to the BDI user profile, details on the actual reasoning process on context knowledge are not given. InforMa is able to process queries that can possibly involve other peers 
and advertise information to these peers in vicinity, relying on graph search and caching techniques but no details were given on how pro-activity is made possible. Another lack identified in the underlying BDI model is that the representation of pre-conditions and effects of intentions are left to the applications, but we have found no clues on how applications fill this issue. Facing an important cost of network transmissions in the exchange process, it seems that this research group is focusing on peer-to-peer networking optimization and trusted exchanges more than on the actual context management. However, they suggested that preparing purpose-driven queries in advance and caching intermediate query results could improve the performance of their system, which is an interesting approach that should be considered in distributed context-aware systems.

The CORBA-based GAIA platform proposed by (Ranganathan, Al-Muhtadi, \& Campbell, 2004) focuses on hybrid reasoning about uncertain context, relying on probabilistic logic, fuzzy logic and Bayesian networks. In their approach, context knowledge is expressed using predicates which classes and properties are defined in a DAML+OIL ontology (Horrocks, 2002). Predicates can be plugged directly into rules and other reasoning and learning mechanisms for handling uncertainty. This choice reduces the overhead of the CoBrA system relying on RDF triples. Rules are processed by the XSB engine [http://xsb.sourceforge.net/], which is described as a kind of optimized Prolog that also supports HiLog, allowing unification on the predicate symbols themselves as well as on their arguments. HiLog's sound and complete proof procedure in first-order logic is needed to write rules about the probabilities of context.

GAIA's authentication mechanism demonstrates the usefulness of fuzzy/uncertain context reasoning. It allows users to authenticate with various means such as passwords, fingerprint sensor or bluetooth phone proximity. Each of these means have different levels of confidence, and some user roles may require that the user authenticates himself on two of them to cumulate their confidence level up to the required level.

Although GAIA proposes a common reasoning framework, application developers have to define the expected context inputs and specify the reasoning mechanism to be used by providing Prolog/HiLog rules (for probabilistic/fuzzy logic) or Bayesian networks. A graphical user interface is provided to help developers construct rules, whereas MSBN (Microsoft's Belief Network) can be used to create Bayesian nets. Although Bayesian networks are a powerful way to perform probabilistic sensor fusion and higher-level context derivation, they need to be trained and inference with large networks (more than 50 nodes) becomes very costly in terms of processing and can result in scalability problems.

Basing on previous works, (Gu et al., 2004) propose SOCAM (Service-Oriented Context-Aware Middleware), another OWL-based context-aware framework with the intention to address more general use cases by adding more qualitative information on acquired context. The classifiedAs property allows categorization of context facts as Sensed, Defined, Aggregated or Deduced. The dependsOn property allows justification of a deduced context based on other context facts. Another contribution is the possibility to qualify context information with parameters such as accuracy, resolution, certainty and freshness. The SOCAM framework was proven (Gu, Pung, \& Zhang, 2004) to reason successfully on uncertain contexts using Bayesian Networks, but no performance results were given. The same group of authors have also carried out a performance experiment of the CONON ontology (Wang, Zhang, Gu, \& Pung, 2004) depicted on Figure 5, which is the name that was given to SOCAM's context ontology. Their results show that the duration of the reasoning process increases exponentially with the number of RDF triples stored in the context knowledge base, which reveals that this approach is not scalable for a widespread context-aware system. Therefore two leads were proposed to increase performance:

- to perform static, complex reasoning tasks (e.g., description logic reasoning for checking inconsistency) in an off-line manner.

- to decouple context processing and context usage, so that context reasoning can be performed by resource-rich devices (such as a server) while the terminals can acquire high- 
level context from a centralized service, instead of performing excessive computation themselves.

Later works of that team were focused on the peer-to-peer architecture for context information systems.

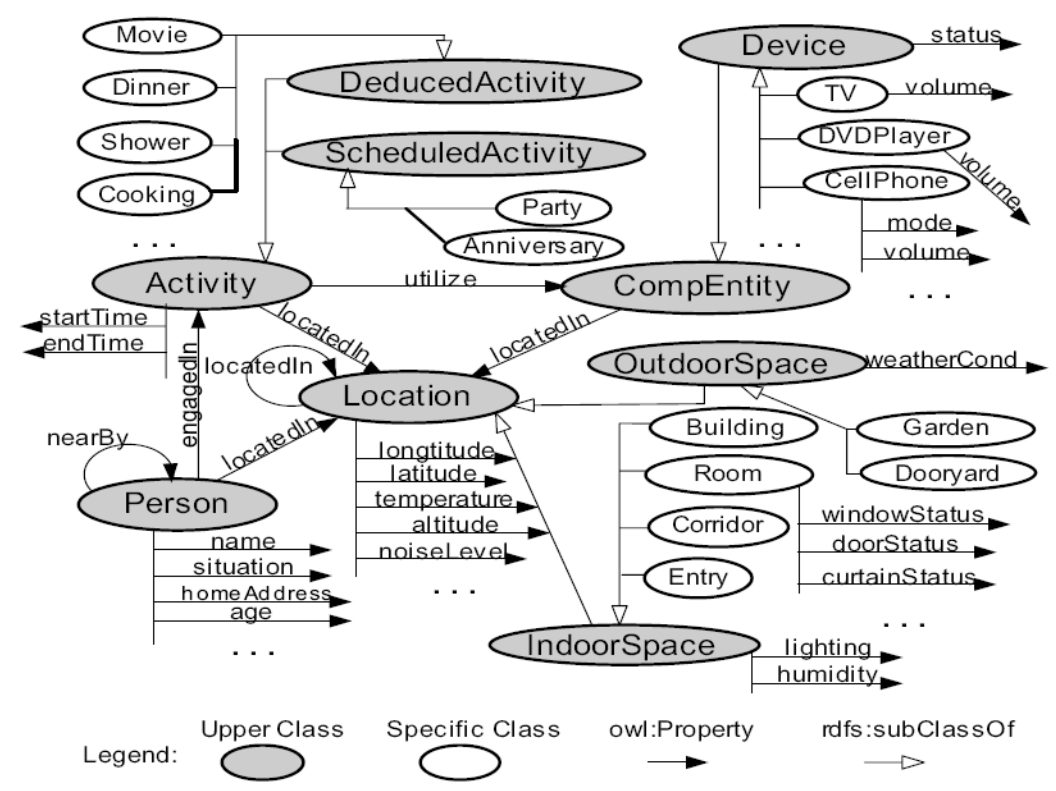

Figure 5: Partial definition of the CONON ontology extended with the home domain (Wang, Zhang, Gu, \& Pung, 2004) $\square$

Basing on the CONON ontology, (Truong, Y. Lee, \& S. Y. Lee, 2005) proposed the PROWL language ("Probabilistic annotated OWL") to generalize fuzzy/probabilistic reasoning from applications to domains by mapping Bayesian Networks to ontology classes and properties. This approach must be experimented with various context-aware applications to prove its feasibility.

The FP6 IST project SPICE (Service Platform for Innovative Communication Environment) brought a fresh approach to ubiquitous system, considering them in a wider scope centered on semantic knowledge management for improved ubiquitous end-user services (SPICE, 2006) (SPICE, 2007). On its Knowledge Management Layer, SPICE proposes two different implementations of the context provisioning subsystem: the IMS Context Enabler (ICE) (M. Strohbach, Bauer, E. Kovacs, C. Villalonga, \& Richter, 2007) and the Knowledge Management Framework (KMF). In ICE, the SIP protocol is leveraged to control the parameters of the exchange sessions (e.g. data sets to communicate, update trigger, update frequency) and to flexibly adjust the communication path based on the changes in network structure and available context information. Both of these implementations rely on a shared ontology called the Mobile Ontology which is freely downloadable on the Internet [http://ontology.ist-spice.org/], the most important difference being the interfaces: ICE uses SIP whereas KMF uses OWL over SOAP Web Services for exchanging context information. However, gateways are also provided so that context data can be converted from a format to the other. Therefore we will abstract these implementations and focus on the common knowledge model. Embracing the recommendations of the W3C, SPICE Mobile Ontology is defined in OWL and the context data is expressed in RDF. Inspired from the Dutch project Freeband Awareness, SPICE's Physical Space ontology has a finer granularity than any previous context ontology: it notably defines properties for connections between rooms and floors. Following the approach of the « Doppelgänger User Modeling System » (Orwant, 1995), SPICE's User Profile ontology supports domain-specific and conditional (situation-specific) submodels. In this approach, the profile contains subsets which are considered on certain conditions expressed with the form: Context Type, Operator, Value. This allows variations of the profile, depending on the user's context 
and/or the targeted application/service.

The Knowledge Management Layer also contains a Knowledge Storage module, a Profile Manager, a Service and Knowledge Push and Notification module and three kinds of Reasoners: a Predictor, a Learner and a Recommender. The reasoners can request past knowledge directly from context sources or from an external knowledge storage source. Both feedback-based and observation-based learning are supported, generating LearntRule and LearntRuleSet instances in OWL. The results can be leveraged to propose Recommendations to the user. Experimental results on the use of different learning techniques are to be published. Another interesting contributions of SPICE in the contextawareness domain is the use of a KnowledgeParameter class that is used to qualify context information with values defining their probability, confidence, timestamp, temporal validity and accuracy. However we have not found any mechanism that is similar to the "dependsOn" property supported by SOCAM to justify high-level context with lower-level facts from which it was inferred.

Another part of the SPICE project called the Distributed Communication Sphere (Kernchen et al., 2007) allows dynamic discovery of user's surrounding devices, networks and services. This part includes components that leverage context knowledge to enable multimodal interaction, content delivery, data synchronization and dynamic widgets on terminals, requiring a lightweight rule engine to be deployed on every terminal. SPICE also provides the End User Studio, an Eclipsebased GUI shown on Figure 6 that allows end users to create custom trigger-action rules visually.

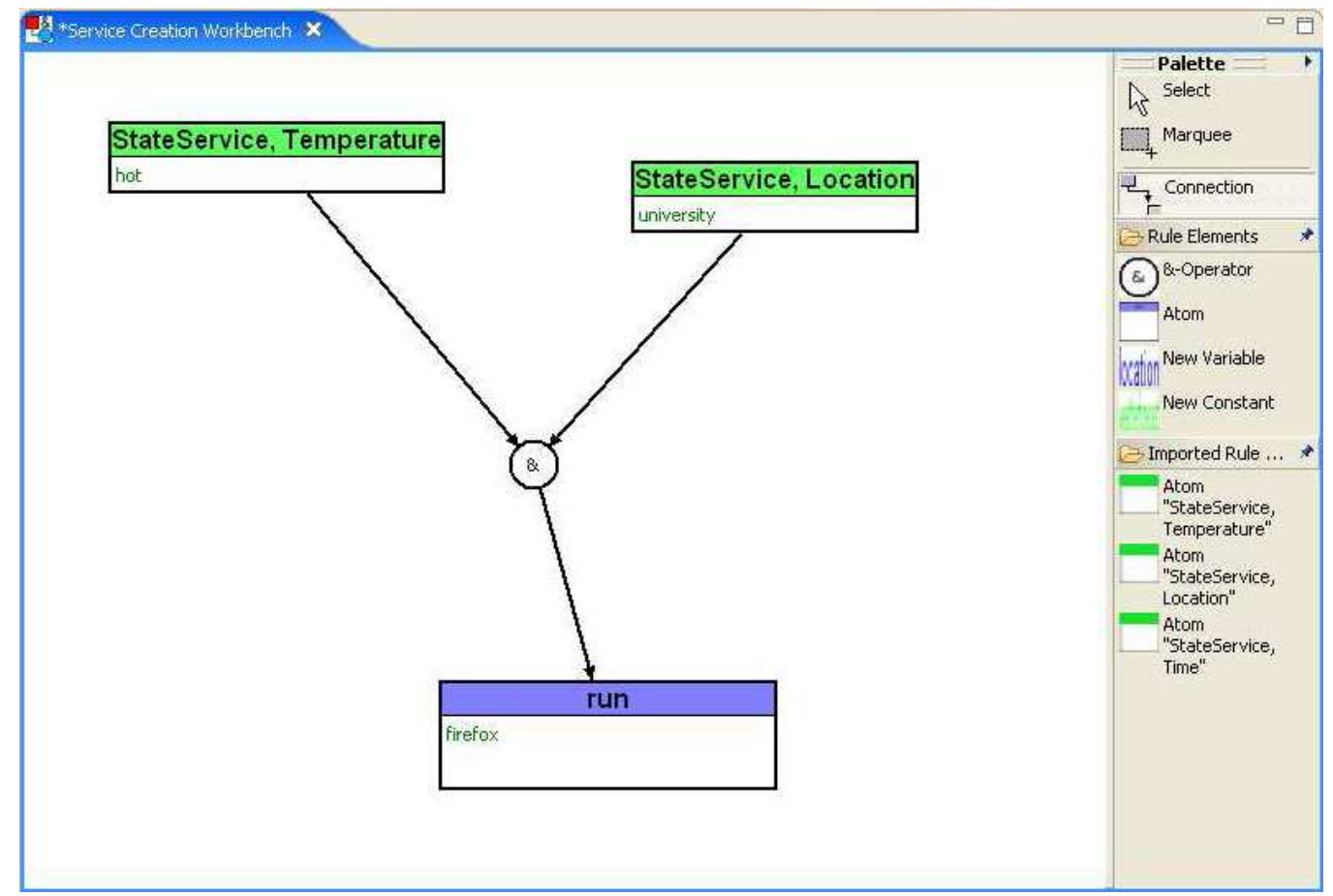

Figure 6: Creating a rule-based service using SPICE's End User Studio (SPICE 2007)

\section{Other approaches and leads}

One of the biggest identified issues in previously reviewed semantic context-aware systems is the processing time required for reasoning on context knowledge. To answer this issue, (Ejigu, Scuturici, \& Brunie, 2007) proposed an hybrid context management and reasoning system (HCoM) which relies on a heuristic-based context selector to filter the context data to be stored in the semantic context base for reasoning, the rest being stored in a relational database, as depicted on Figure 7. They report that this approach is more scalable than pure semantic context-awareness 
systems when the number of static context instances increases. (Lin, Li, Yang, \& Shi, 2005) propose a similar approach but they filter context data according to their relevance to running applications instead of usage heuristics, in order to boost the reasoning performance.

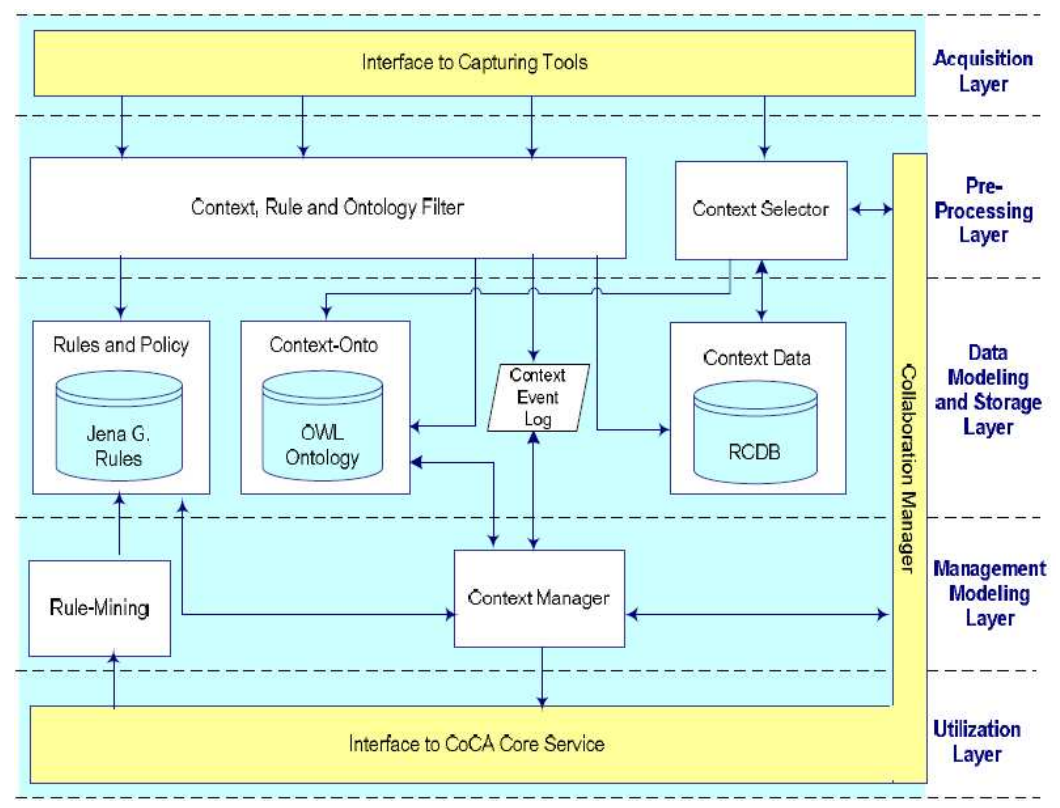

Figure 7: HCoM: Hybrid Context Management and reasoning system (Ejigu et al. 2007)

(Tan, Zhang, Wang, \& Cheng, 2005) propose to move from on-demand context reasoning to eventdriven context interpretation so that reasoning on context data is processed as soon as it is received by the context management framework. However, in their distributed system, the performance is reduced because of increased communication overheads. Moreover, it does not support uncertainty yet.

Hybrid context management approaches leverage the assets of both relational and semantic context management, therefore they should be considered in the aim of building a powerful and scalable context-awareness system. Nevertheless, the selection/filtering of context data to be merged in the semantic database is not trivial and may need further research.

\section{Semantics for adapted human-system interactions}

After context-awareness, another key aspect of Ambient Intelligence is how the user interacts with the digital world. Today, we are still using computers. Whereas most people carry their own powerful mobile phone with them, most of the content and services that we like to use are not adapted to general mobile devices with their constraints (small screen, no keyboard...). Of course some of those have been adapted specifically to some popular platforms like the iPhone, but the vision of Ambient Intelligence is to bring most of them to virtually any terminal, according to its capabilities. Therefore, ambient services need to know the capabilities of every platform it's being used on, and they need to adapt the user interface according to these capabilities. In this section we will review existing technologies for the discovery of devices and the description of their capabilities in order to enable rich user interactions and multimodality.

\section{Semantic discovery and description of interfaces}

CC/PP (Composite Capabilities / Preferences Profile) (Klyne et al., 2004) is a recommendation from the W3C based on the Resource Description Framework (RDF) to create profiles that describe device capabilities and user preferences. It provides a syntax and tools to create terminal profiles 
and preference vocabularies, and thus can not be used as is. Indeed, the vocabulary of capabilities to use for defining profiles are not in the scope of this recommendation and only structural rules and guidelines for interoperability are provided. However, the recommendation includes an pointer to the UAProf vocabulary as a referred example; we will review this vocabulary below. Among the features of the CC/PP syntax, allowed value types are listed, and the definition of default values is explained. The state-of-the-art of (SPICE, 2006) pointed out that conditional constraints are not supported in CC/PP. Moreover, the recommendation clearly informs that a CC/PP profile may include sensitive data, and delegates the enforcement of privacy to the application/system.

UAProf (User Agent Profile) (WAP Forum, 2001) is a CC/PP vocabulary for WAP enabled cell phones developed by the Open Mobile Alliance (OMA). The idea is that compliant cell phones have their capabilities described in a profile stored on a web repository so that adaptive services can gather this information in order to tailor content for embedded web browsers. This vocabulary is focused on software and hardware capabilities, and thus does not cover preferences.

WURFL (Wireless Universal Resource File) [http://wurfl.sourceforge.net/uaprof.php] is a collaborative effort to build an open XML file that describes device profiles based on fixes of their UAProf profiles. This promising initiative addresses several shortcomings of the original UAProf approach in which profiles can be inconsistent across providers, not up to date, or even do not exist.

The Foundation for Intelligent Physical Agents (FIPA) also proposed a device description ontology (FIPA, 2002) that can be used to reason and make decisions on the best device and modalities to create a user interface in multi-agent systems. Due to the nature of multi-agent systems, this approach differs from CC/PP in the manner of transmitting the profile. Instead of providing its complete profile on-demand, the terminal returns profile subsets adaptively to requests, allowing to set the granularity and scope of the required profile content in a gradual negotiation between agents. Whereas a CC/PP profile defines the capabilities for the software, hardware and the browser, FIPA Device Description supports the description of agent-related capabilities instead of the browser's. However, it is possible to use this ontology in a CC/PP profile, similarly to UAProf.

Even though this approach is not based on semantic technologies, the UPnP (Universal Plug and Play) discovery protocol (UPnP Forum, 2003) defines a XML language that can describe a physical device into a hierarchy of logical devices which map every hardware component of the device and thus its corresponding capability. The modularity of this approach is interesting and should be considered in order to improve re-usability of profiles, according to the fact that common hardware components are part of many devices.

\section{Semantics for multimodality}

When devices and their capabilities are discovered, their use for multimodal interaction requires additional negotiation and synchronization so that user interaction constraints are respected for a rich user experience. The constraints to validate cover the quality of rendering/sampling, the robustness of the connectivity, the privacy of exchanges (e.g. displaying emails on a public screen should be avoided), and also the environmental context and user preferences.

The members of the W3C Multimodal Interaction Working Group propose their specifications of a Multimodal Interaction Framework (W3C, 2003) based on a central Interaction Manager that connects user inputs (e.g. audio, speech, handwriting, keyboard...) and outputs (e.g. speech, text, graphics, motion...) to applications and two other components, as shown on Figure 8:

- The Session Component, which handles the state management for application sessions that may involve multiple steps, multiple modality modes, multiple devices and/or multiple users.

- The System and Environment component, which handles the changes of device capabilities, user preferences and contextual/environmental conditions. 
The Interaction Manager coordinates data and manages the execution flow from various input and output modality components. It is responsible to combine various user inputs for submitting meaningful actions to applications (multimodal fusion) and to dispatch responses to the user through various output interfaces (multimodal fission).

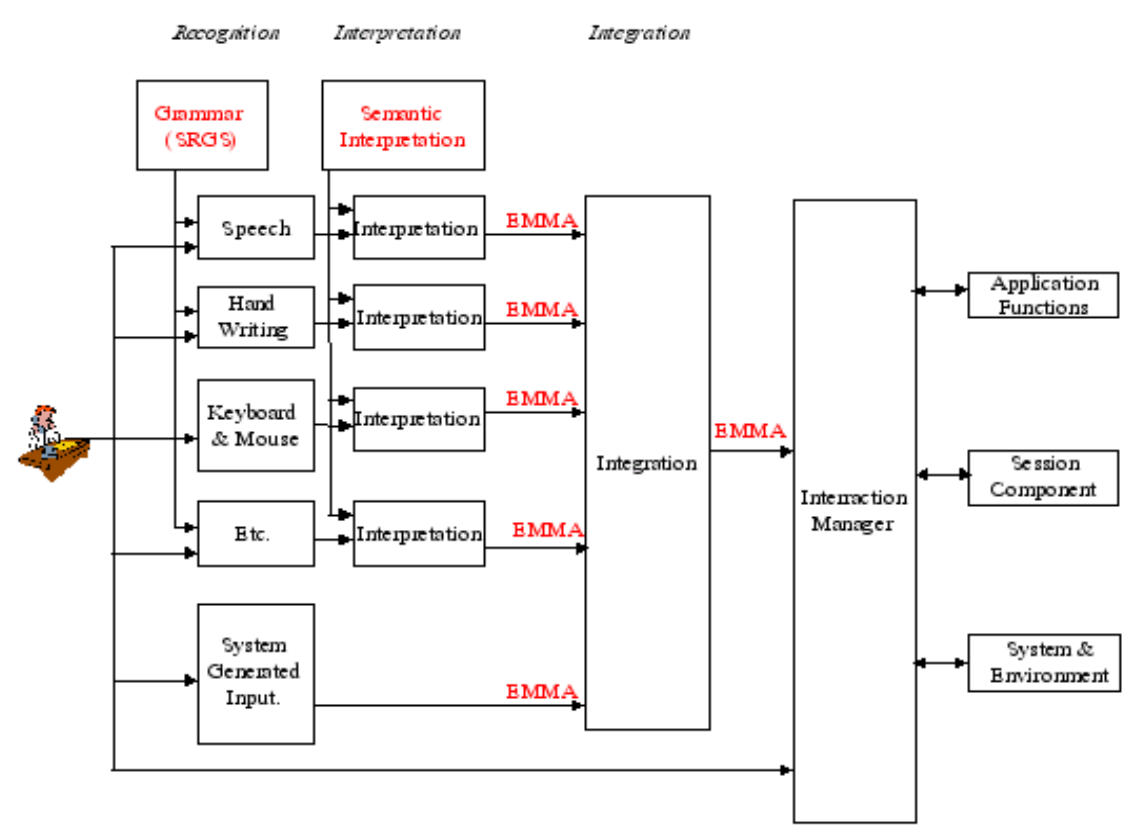

Figure 8: The input process of the Multimodal Interaction

Framework (W3C 2003)

Also proposed by the W3C Multimodal Interaction Working Group, the EMMA (Extensible MultiModal Annotation) markup language (W3C, 2007) is a XML markup language for describing the interpretation of user inputs. An example of input interpretation is transcription into words of a raw signal, for instance derived from speech, pen or keystroke input, or a set of attribute/value pairs describing a gesture. The interpretations of user's input are expected to be generated by signal interpretation processors, such as speech and ink recognition, semantic interpreters, and other types of processors for use by components that act on the user's inputs such as interaction managers. As shown on Figure 8, user inputs are processed in two layers to generate EMMA data which is integrated for submission to the Interaction Manager. The two layers of input processing consist of:

- Recognition components, which capture natural input from the user and translate them into a form useful for later processing. (e.g. speech to text, handwritten symbols and messages to text, mouse movements to $\mathrm{x}-\mathrm{y}$ coordinates on a two-dimensional surface...)

- Interpretation components, which further process the results of recognition components by identifying the meaning/semantics intended by the user. (e.g. pointing somewhere on a map would result in knowing the name of the corresponding country, nodding or saying "I agree" would both mean acceptation from the user...)

Recommended by the $\mathrm{W} 3 \mathrm{C}$, EMMA is probably going to become a standard for annotation of multimodal inputs. It has shown its usefulness especially for speech-based dialog in extensible multimodal applications (Reithinger \& Sonntag, 2005; Manchón, del Solar, de Amores, \& Pérez, 2006; Oberle et al., 2006).

The IST project Mobilife proposed a solution (Kernchen, Boussard, Moessner, \& Mrohs, 2006) to describe devices and modality services to form context-aware multimodal user interfaces. Their 
identified requirements include the deployment of a fission component implementing a rule-based algorithm on the device in order to adapt the user's mobile multimodal interface best to the current situation. In the SPICE project (Kernchen et al., 2007), the «Multimedia Delivery and Control System » depicted on Figure 9 has been developed as a part of the "Distributed Communication Sphere ", is a multimodal platform relying on the W3C-recommended Synchronized Multimedia Integration Language (SMIL) (Ayars et al., 2000), that supports multimodal fusion and fission. First, the " resource discovery system » of the MDCS finds appropriate interfaces, then modalities are selected according to user preferences, context (e.g. Walking, driving...), available resources in user's DCS and provision constraints. Modality, device and network recommendations are proposed by the knowledge management framework. This implementation is available as an open source project [https://sourceforge.net/projects/mdcs].

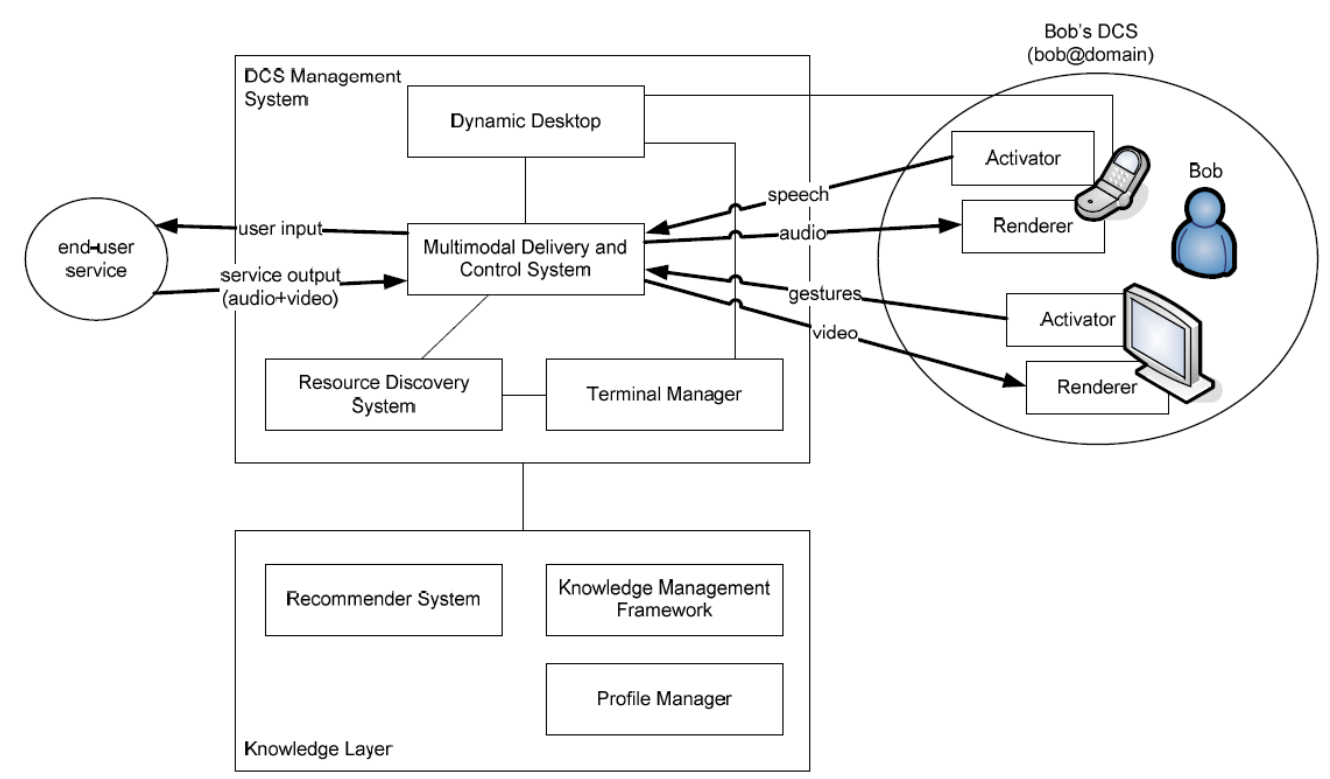

Figure 9: SPICE Multimedia Delivery and Control System (Kernchen et al. 2007)

Today, semantic technologies have shown their usefulness to improve the discovery, description and exploitation of multimodal interfaces. However, the vision of ubiquitous computing in which any screen can be used to display personal information requires privacy enforcement mechanisms, especially if public screens are expected to be shared as well for this matter. In the next part, we will study how semantic technologies can help to enforce privacy in such systems.

\section{Semantics for privacy}

The vision of ubiquitous computing in which personal information flows in a highly networked ecosystem requires privacy enforcement mechanisms, especially if public screens are expected to be shared for displaying such information as well as personal terminals. In this part, we will study how semantic technologies can help to enforce privacy in ubiquitous systems.

According to (Damianou, Dulay, Lupu, \& Sloman, 2001), the use of policies is an emerging technique for controlling and adjusting the low-level system behaviors by specifying high-level rules. Policies enforced using semantic rule engines are implemented in most secure semantic context-aware platforms studied earlier in this chapter. In their review of semantic web languages for policy representation and reasoning, (Tonti et al., 2003) explain that "the use of policies allows administrators to modify system behavior without changing source code or requiring the consent or cooperation of the components being governed". KAoS and Rei are both semantic policy languages: KAoS is an OWL-based language and uses Java Theorem Prover to support reasoning whereas Rei uses Prolog and RDF-S. They also propose different enforcement mechanisms: KAoS 
requires to the enforcers to be implemented and integrated in the system entities to control, whereas the Rei's actions are to be executed outside the Rei's engine.

(Shankar \& Campbell, 2005) propose an extension to the ECA (Event-Condition-Action) rule framework, called Event-Condition-PreCondition-Action-PostCondition (ECPAP). In this framework, actions are annotated with axiomatic specifications that enable powerful reasoning to detect conflicts and cycles in policies.

(Brar \& Kay, 2004) propose "secure persona exchange" (SPE), a framework based on W3C's Platform for Privacy Preferences (P3P) for secure anonymous/pseudonymous personal data exchange. This framework allows users to negotiate agreements with services that declare their privacy practices and request personal data. The P3P defines such a semantic service description format whereas privacy preferences are described using the APPEL language (A P3P Preference Exchange Language). SPE addresses the following identified end-user requirements: purpose specification, openness, simple and appropriate controls, limited data retention, pseudonymous interaction and decentralized control.

We have identified three semantic models that can be used to enforce privacy in ubiquitous systems: rule-based policies, ECA-based policies and secure exchange negotiation according to privacy preferences. Although the last one is the only to address the issue of secure exchange of personal information, these models have yet to be compared in a common experiment implementing all the requirements of a complete Ambient Intelligence system.

\section{Semantics for the social communications and activities}

At the era of the social networking and participative web, of always-connected chat messengers and virtual worlds, people communicate and exchange more and more over the Internet. If we expect computers to disappear, we need to adapt the communication and exchange paradigms to take the context of the users into account and to leverage the social knowledge held in web platforms in order to improve the intelligence of context-aware systems. One of the key points of such communications is user presence, because being online does not mean being attentive to any discussion anytime. The second point that we will discuss covers the user profiling techniques and expression of the social graph. Finally, promising technologies for maintaining connected social activities in an interoperable way will be discussed.

\section{User presence and communication}

An important part of the context information in a communication network is presence, which is information on reachability, availability, and status across all communication channels (e.g., networks, applications, transports over Internet, wireless and wireline).

Two major presence exchange formats are considered here. The first one is SIMPLE (SIP for Instant Messaging and Presence Leveraging Extensions), an extension of the SIP protocol recommended by the Open Mobile Alliance (OMA) that adds new features such as: voice, video, application sharing, and messaging. Leveraging the communication and security of the IMS platform, SIMPLE permits the user's presence to take into account his willingness, ability and desire to communicate across all different kinds of media types, devices, and places. Even though it is not a semantic language, the Dutch project Freeband Awareness (Bargh et al., 2005) chose the SIP/SIMPLE protocol for realizing a context-aware network infrastructure with the focus on secure and privacy-sensitive context exchange between a core network owner (e.g. a cell carrier) and external entities. In other projects, SIP utilization can be limited to exchanges that imply an interaction with the user: notifications, confirmations... In the SPICE project (M. Strohbach, E. Kovacs, \& Goix, 2007), SIP is used to share presence information with the IMS platform and exchange data with the communicating user. SPICE's Mobile Ontology includes a presence ontology based on PIDF (Presence Information Data Format) which allows definitions of the user's 
input, mood, contact relationship, place characteristics, current activity, and service. Transformation templates are provided to switch from the internal RDF representation into PIDF, and the other way round.

SIP has a wide range of possible uses but is not an optimal solution for all kinds of exchange. (Houri, 2007) complained the weakness of SIP/SIMPLE in domain scaling. Furthermore it appears (Saint-Andre, 2005) that SIP/SIMPLE does not support advanced messaging mechanisms like workflow forms, multiple recipients, reliable delivery and publish-subscribe which are useful for context-aware systems. PIDF has shown to be suitable for the SPICE project.

\section{Profiling and social graph}

Considering the user's profile and social graph is important to personalize access to information and communication means. At a time where silo web-based social networks are exploding, many initiatives try to free our social data from these platforms using interoperable formats.

FOAF (Friend-of-a-Friend) (Brickley \& Miller, 2007) is a RDF vocabulary based on an OWL ontology to describe people profiles, friends, affiliations, creations etc... FOAF's vision is a decentralized and extensible machine-readable social network based on personal profiles. The profile contains descriptions of personal user data, possibly his/her work history, and links to his contacts and affiliated services. Each person has a unique identifier, usually a hash of the email address. The community of FOAF users being principally made of researchers and semantic web enthusiasts, it does not compete with popular social networks like LinkedIn [http://www.linkedin.com], Myspace [http://www.myspace.com] or Facebook [http://www.facebook.com]. However, many tools have appeared, including FOAFexplorer [http://xml.mfd-consult.dk/foaf/explorer/] which can be used to visualize FOAF profiles. However, there is a potential privacy issue with this language because selective privacy-aware views of a FOAF file are not addressed. It may be interesting to evaluate a mechanism similar to the conditional profiles utilized in the SPICE project or to enforce selective distribution of content using a policy-based system.

SIOC (Semantically-Interlinked Online Communities, http://sioc-project.org) represented on Figure 10 is an ontology-based framework aimed at interconnecting online community sites and internetbased discussions. The idea is to enable cross-platform interoperability so that conversation spanning over multiple online media (e.g. blogs, forums, mailing lists...) can be unified into one open format. The interchange format expresses the information contained both explicitly and implicitly in internet discussion methods, in a machine-readable manner. A similar approach is proposed by the OPSN (Open Portable Social Network, http://www.opsn.net/) initiative which also covers notification and synchronization of contacts across platforms. However there is no existing implementation, and privacy control for personal published information seems not to have been addressed yet. DISO (distributed social networking, http://diso-project.org/), is yet another collaborative work to follow. 


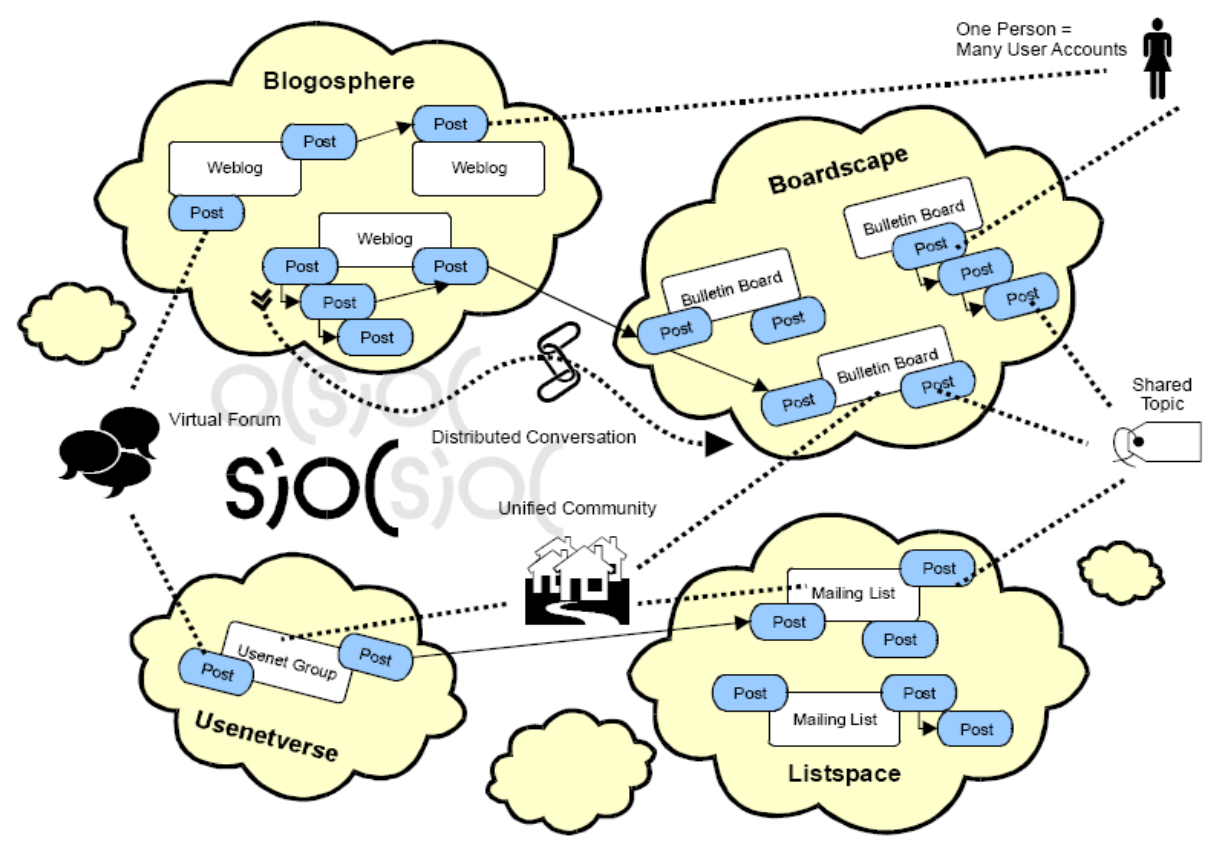

Figure 10: Overview of SIOC: Semantically-Interlinked Online

Communities [http://sioc-project.org]

These initiatives would be a promising way to leverage consistent social relations, discussions and exchanges from various web platforms in order to build a more precise profile of user's interests, like with the APML language (Attention Profiling Mark-up Language, http://www.apml.org/), and qualify the types of relations in order to improve the social communication experience.

\section{Social interactivity}

With its open application platform, the social networking site Facebook became a huge Internet player in a few months, attracting many service providers and increasing their population of users significantly. Indeed, Facebook made it easy for application developers to leverage the user's profile and social graph of the underlying platform, and thus bring user-friendly services with a social dimension. For example, as shown on Figure 11, the "Movies" application allows the user to rate movies so that his/her favorite movies are shown on his profile page. But the most interesting aspect of this application is the possibility for friends to compare their movie tastes to evaluate their compatibility. 


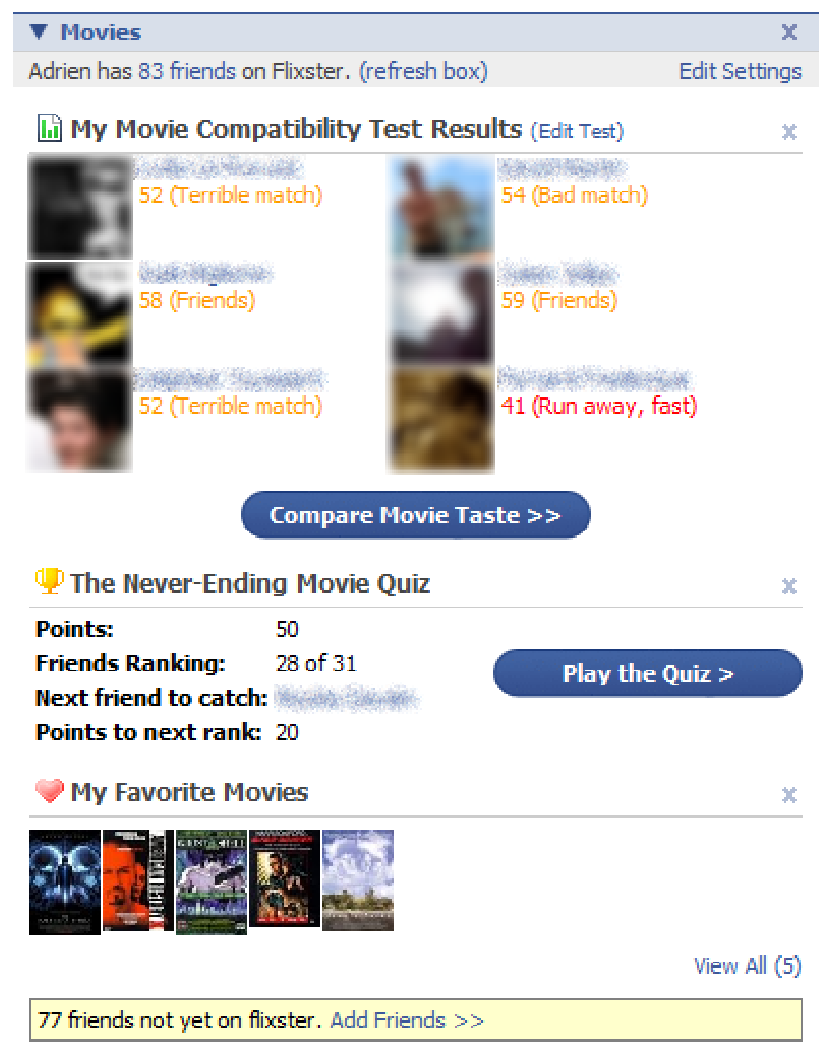

Figure 11: The "Movies" application on

Facebook

Because there are many existing social networking sites on the Internet that are adopting the application platform approach à la Facebook, Google initiated the OpenSocial project, an interoperable framework to build applications on any compliant social networking site. However this framework implements basic contact management actions only and don't have access to all the information and capabilities of all social networking sites. For example, some of them are capable of exchanging "pokes", "gifts" and comments, but there is no interoperable way of invoking these capabilities from OpenSocial so far. This could be the opportunity to develop an ontology of social interaction which could be enriched by the platforms and gradually supported by applications without preventing them to work in degraded mode (e.g. by sending a comment instead of a gift, if this capability is not supported by the platform).

The possible links between ubiquitous context-aware platforms and existing "Web 2.0" platforms (O'Reilly, 2005) which hold valuable knowledge about people's lives and support applications/services that empower rich communication activities have been neglected by academia, and Internet players are working together to build controlled interoperability. Although extraction of consistent knowledge from the Web 2.0 is not trivial (Gruber, 2006), there is a huge value in social networks and user-generated content that should be leveraged to extend the possibilities of Ambient Intelligence, therefore it is important for researchers to get involved in the ongoing initiatives for interoperability of social platforms.

\section{Future trends}

We have depicted an overview of several past and current semantically-powered approaches of context-aware systems, adapted human-system interactions, privacy enforcement and social communications and activities. We will now propose several research leads towards Social Ambient Intelligence based on semantic technology. 


\section{Technical issues}

For every research domain covered in this chapter, several open issues were identified and the approaches are yet to be benchmarked on a common scenario to evaluate their respective assets and lacks. A major challenge is scalability of semantic rule-based systems (which are employed in context-aware systems, privacy policy enforcement systems and several multimodal systems). The reasoning process needed for prediction (and thus pro-activity) is very complex and usually requires a processing power that is exponentially proportional to the size of the knowledge base, which is not acceptable for a widescaled ubiquitous system. In some cases, an additional overhead was caused by transfers of facts from a knowledge base to the rule engine. Therefore, closer coupling or integration of rule engines with knowledge bases is to be considered, as well as hybrid knowledge management approaches based on filtering of data to be taken in account by the reasoner.

\section{Converging with the social web}

We also pointed out that state-of-the-art ubiquitous platforms should leverage the existing web platforms, or at least inspire from them. It is time for the social web, context awareness, and multimodal interfaces to converge into an ambient platform that enforces privacy. We believe that the semantic technologies are the best enablers for interoperability, extensibility and intelligent exploitation of user, hardware and social web knowledge in order to improve interactions between users and information. However, leveraging web knowledge in a semantic ubiquitous system may not be a trivial task according to (Strassner et al., 2007) who claimed that, in order for ontologies to be adopted by a system, this system should have a sufficient amount of semantic knowledge and minimal legacy information to carry. Indeed, the Semantic Web still being an unachieved vision (Berners-Lee, Hendler, \& O. Lassila, 2001; Cardoso, 2007), most websites don't rely on semantic technologies to maintain their data. We have presented several initiatives that intend to create interoperable standards based on semantic technologies for universal exploitation of user-generated content and communications kept in separate web platforms. Academics should get involved in this process, in order to take into account the requirements of Social Ambient Intelligence platforms that will leverage these standards. In the mean time, web platforms APIs can be used to build gateways between specific web social platforms and ubiquitous systems. For example, user feeds (e.g. Facebook's mini-feed, twitter, del.icio.us...) could be analyzed as an additional source of context knowledge in the aim of identifying user activities and profile. On the other hand, ubiquitous systems could also be used to push content to these platforms, e.g. automatic presence information inferred from the context.

\section{Bringing ubiquitous systems to people}

Another issue that we want to address here is the lack of integration and public visibility of research works related to Ambient Intelligence. The growing ubiquity of networks (infrastructures and adhoc), screens and mobile devices brings more exciting opportunities for people to communicate and exchange content but we lack interoperability standards. Where is the unified platform that allows me to use my mobile phone as a remote control for all my domestic and multimedia appliances ? When will the current music playlist being played on my computer be automatically transferred to my portable MP3 player before I leave home ? Most context-aware systems are still far from Weiser's vision of Ubiquitous Computing. In the meantime, innovative ubiquitous products appear on the market, such as electronic photo frames, widget displays, toys that can give weather reports and read emails, and powerful domestic management systems but they all work on their own because we lack common standards and platforms. One way of making people progressively adopt ubiquitous systems is to advertise them as applications on popular social platforms (e.g. Facebook), inviting users to deploy required software on their terminal to benefit from exciting services that could possibly leverage users' context. Some people may be reluctant to use such systems at first, but we believe that there are solutions to make them accept it. 


\section{Gain trust from potential users}

Potential users of ubiquitous context-aware systems can be reluctant for the following reasons:

1. Privacy

Users will be concerned with the idea of provisioning private contextual knowledge (such as user positioning) to a "black-box" system which they may not trust, because they are afraid of loosing control of this information, of being tracked or even spied. Moreover, most Internet users are already concerned with spam, and many already complain about profiling operated by web sites to improve the relevance of advertising; therefore sharing contextual knowledge can be seen as a major threat for privacy and control of personal information. We believe that advertising should be taken in account as the fair counterpart of a service, but it must be moderated by the system. E.g. a music recommendation service that advertises live performances and merchandising of one's favorite artists seems like an equitable service that profit both to the user and the service provider. Nevertheless, the user must constantly be in control of his private information and confidentiality/security of exchanges must be enforced using mechanisms such as pseudonymity or cryptology. Transparency of the ubiquitous system's implementation and knowledge base can be a major source of trust for users, like it has been with open source software.

2. Intrusion

Subscription to many services that have access to extensive knowledge about users (e.g. their interests, their social network) and also privacy policy management can lead to digital pollution. Users could receive hundreds of recommendations, being asked hundreds of questions about their current situations and confirmations for proposed relevant actions to undertake. Research must be carried out to moderate explicit user interaction (i.e. requests and notifications) without compromising intended communications, user awareness and control. A promising approach for semi-autonomous control of user private data is the use of policies. However, as (O. Lassila, 2005) pointed out, we need a rich representation of policies so that users can define and visualize their privacy rules in a clear and easy way, and delegate their enforcement to the system.

\section{Conclusion}

In this chapter, we have reviewed several utilizations of semantic technologies for context management, adaptive human-system interaction, privacy enforcement and social communications in the scope of Ambient Intelligence. Based on identified benefits and lacks, we proposed several research leads towards the realization of Social Ambient Intelligence based on the social web and semantic technologies. Through our involvement in several ongoing European research projects and national initiatives, including EASY Interactions [http://www.itea2-easy-interactions.org/], SPICE [http://www.ist-spice.org/], m:Ciudad [http://www.mciudad.org/index ing.htm] and Villes 2.0 [http://www.villes2.fr/], we will strive to focus our research on these points and to convey our position and trends to our collaborators.

\section{References}

Ayars, J., Bulterman, D., Cohen, A., Day, K., Hodge, E., Hoschka, P., et al. (2000). Synchronized multimedia integration language (smil) 2.0 specification, Work in progress. W3C Working Drafts are available at http://www. w3. org/TR, 21.

Baldauf, M., Dustdar, S., \& Rosenberg, F. (2007). A survey on context-aware systems, International Journal of Ad Hoc and Ubiquitous Computing, 2(4), 263-277. 
Bargh, M., Benz, H., Brok, J., Heijenk, G., Groot, S. H. D., Peddemors, A., et al. (2005, January 10). Initial architecture for awareness network layer J. Brok (Ed.). Retrieved from http://awareness.freeband.nl.

Berners-Lee, T., Hendler, J., \& Lassila, O. (2001). The semantic web, Scientific American, 284(5), 28-37.

Brar, A., \& Kay, J. (2004). Privacy and security in ubiquitous personalized applications.

Brickley, D., \& Miller, L. (2007, November 2). Foaf vocabulary specification, Namespace Document. Retrieved from http://xmlns.com/foaf/spec/.

Cardoso, J. (2007). The semantic web vision: where are we?, Intelligent Systems, 22(5), 84-88.

Chen, H., Finin, T., Joshi, A., Kagal, L., Perich, F., \& Chakraborty, D. (2004). Intelligent agents meet the semantic web in smart spaces, IEEE Internet Computing, 8(6), 69-79.

Chen, H., Perich, F., Finin, T., \& Joshi, A. (2004). Soupa: standard ontology for ubiquitous and pervasive applications, Mobile and Ubiquitous Systems: Networking and Services, 2004. MOBIQUITOUS 2004. The First Annual International Conference on, 258-267.

Damianou, N., Dulay, N., Lupu, E., \& Sloman, M. (2001). The ponder policy specification language, Policies for Distributed Systems and Networks: International Workshop, Policy 2001, Bristol, Uk, January 29-31, 2001: Proceedings.

Dean, M., \& Schreiber, G. (2004). Owl web ontology language reference, W3C Recommendation.

Decker, S., Erdmann, M., Fensel, D., \& Studer, R. (1999). Ontobroker: ontology based access to distributed and semi-structured information, Database Semantics: Semantic Issues in Multimedia Systems, 351-369.

Dey, A. K. (2001). Understanding and using context, Personal and Ubiquitous Computing, 5(1), 47.

Ejigu, D., Scuturici, V., \& Brunie, L. (2007). Semantic approach to context management and reasoning in ubiquitous context-aware systems In , The Second IEEE International Conference on Digital Information Management(ICDIM 2007), Proceedings of ICDIM'07. (pp. 500-5005). Retrieved from http://liris.cnrs.fr/publis/?id=3242.

FIPA. (2002, December 6). Fipa device ontology specification. 
Gruber, T. (2006). Where the social web meets the semantic web, Lecture Notes in Computer Science, 4273, 994.

Gu, T., Pung, H. K., \& Zhang, D. Q. (2004). A bayesian approach for dealing with uncertain contexts, Proceedings of the Second International Conference on Pervasive Computing.

Gu, T., Wang, X. H., Pung, H. K., \& Zhang, D. Q. (2004). An ontology-based context model in intelligent environments, Proceedings of Communication Networks and Distributed Systems Modeling and Simulation Conference, 2004.

Horrocks, I. (2002). Daml+oil: a description logic for the semantic web, IEEE Data Engineering Bulletin, 25(1), 4-9.

Houri, A. (2007, October 29). Draft-ietf-simple-interdomain-scaling-analysis-02 - presence interdomain scaling analysis for sip/simple, Presence Interdomain Scaling Analysis for SIP/SIMPLE. Retrieved November 13, 2007, from http://tools.ietf.org/html/draft-ietfsimple-interdomain-scaling-analysis- 02 .

Kagal, L. F., \& Joshi, T. A. (2003). A policy language for a pervasive computing environment, Policies for Distributed Systems and Networks, 2003. Proceedings. POLICY 2003. IEEE 4th International Workshop on, 63-74.

Kernchen, R., Boussard, M., Hesselman, C., Villalonga, C., Clavier, E., Zhdanova, A. V., et al. (2007). Managing personal communication environments in next generation service platforms, Mobile and Wireless Communications Summit, 2007. 16th IST, 1-5.

Kernchen, R., Boussard, M., Moessner, K., \& Mrohs, B. (2006). Device description for mobile multimodal interfaces In . Mykonos, Greece.

Klyne, G., Reynolds, F., Woodrow, C., Ohto, H., Hjelm, J., Butler, M. H., et al. (2004). Composite capability/preference profiles (cc/pp): structure and vocabularies 1.0. w3c recommendation, w3c, january 2004.

Lassila, O., \& Khushraj, D. (2005). Contextualizing applications via semantic middleware In , Mobile and Ubiquitous Systems: Networking and Services, 2005 (pp. 183-189).

Lassila, O. (2005, August). Using the semantic web in mobile and ubiquitous computing. Jyväskylä (Finland).

Lin, X., Li, S., Yang, Z., \& Shi, W. (2005). Application-oriented context modeling and reasoning in pervasive computing, Proceedings of the The Fifth International Conference on Computer 
and Information Technology, 495-501.

Manchón, P., del Solar, C., de Amores, G., \& Pérez, G. (2006). The mimus corpus In (pp. 56-59). Genoa, Italy.

Oberle, D., Ankolekar, A., Hitzler, P., Cimiano, P., Sintek, M., Kiesel, M., et al. (2006). Dolce ergo sumo: on foundational and domain models in swinto (smartweb integrated ontology), Submission to Journal of Web Semantics (2006).

O'Reilly, T. (2005). What is web 2.0: design patterns and business models for the next generation of software, O'Reilly. Retrieved from http://www.oreillynet.com/pub/a/oreilly/tim/news/2005/09/30/what-is-web-20.html.

Orwant, L. (1995). Heterogeneous learning in the doppelganger user modelling system, User Modelling and User Adapted Interaction, 4(2), 107-130.

Perich, F., Avancha, S., Chakraborty, D., Joshi, A., \& Yesha, Y. (2005). Profile driven data management for pervasive environments. Springer.

Ranganathan, A., Al-Muhtadi, J., \& Campbell, R. H. (2004). Reasoning about uncertain contexts in pervasive computing environments, Pervasive Computing, IEEE, 3(2), 62-70.

Reithinger, N., \& Sonntag, D. (2005). An integration framework for a mobile multimodal dialogue system accessing the semantic web, Ninth European Conference on Speech Communication and Technology.

Saint-Andre, P. (2005, December 8). Xmpp-simple feature comparison, XMPP-SIMPLE Feature Comparison. Retrieved November 13, 2007, from http://www.jabber.org/protocol/xmppsimple.shtml.

Shankar, C., \& Campbell, R. (2005). A policy-based management framework for pervasive systems using axiomatized rule-actions, Network Computing and Applications, Fourth IEEE International Symposium on, 255-258.

SPICE. (2006). Spice d4.1: ontology definition of user profiles, knowledge information and services. Retrieved February 13, 2008, from http://www.ist-spice.org/documents/D4.1final.pdf.

SPICE. (2007). Spice unified architecture. Retrieved from http://www.istspice.org/documents/SPICE_WP1_unified_architecture_Phase\%202.pdf.

Strang, T., \& Linnhoff-Popien, C. (2004). A context modeling survey, Workshop on Advanced 
Context Modelling, Reasoning and Management, UbiComp, 34-41.

Strang, T., Linnhoff-Popien, C., \& Frank, K. (2003). Cool: a context ontology language to enable contextual interoperability, Distributed Applications and Interoperable Systems: 4th Ifip Wg6. 1 International Conference, Dais 2003, Paris, France, November 17-21, 2003, Proceedings.

Strassner, J., O'Sullivan, D., \& Lewis, D. (2007). Ontologies in the engineering of management and autonomic systems: a reality check, Journal of Network and Systems Management, 15(1), 511 .

Streitz, N., \& Nixon, P. (2005). The disappearing computer, Communications of the ACM, 48(3), 32-35.

Strohbach, M., Kovacs, E., \& Goix, L. W. (2007). Integrating ims presence information in a service oriented architecture.

Strohbach, M., Bauer, M., Kovacs, E., Villalonga, C., \& Richter, N. (2007). Context sessions: a novel approach for scalable context management in ngn networks In . Newport Beach, California, USA.

Tan, J. G., Zhang, D., Wang, X., \& Cheng, H. S. (2005). Enhancing semantic spaces with eventdriven context interpretation, Pervasive Computing: Third International Conference, Pervasive 2005, Munich, Germany, May 8-13, 2005, Proceedings.

Tonti, G., Bradshaw, J. M., Jeffers, R., Montanari, R., Suri, N., \& Uszok, A. (2003). Semantic web languages for policy representation and reasoning: a comparison of kaos, rei, and ponder, The Semantic Web-ISWC, 419-437.

Truong, B. A., Lee, Y., \& Lee, S. Y. (2005). A unified context model: bringing probabilistic models to context ontology, Lecture notes in computer science, 566-575.

UPnP Forum. (2003, December 2). Upnp device architecture 1.0. Retrieved from http://www.upnp.org/.

W3C. (2003). W3c multimodal interaction framework, W3C Note. Retrieved from http://www.w3.org/TR/mmi-framework/.

W3C. (2007). Emma: extensible multimodal annotation markup language, W3C Working Draft 14 December 2004. Retrieved from http://www.w3.org/TR/emma/.

Wang, X., Zhang, D., Gu, T., \& Pung, H. (2004). Ontology based context modeling and reasoning 
using owl In , Pervasive Computing and Communications Workshops, 2004. Proceedings of the Second IEEE Annual Conference on (pp. 18-22).

WAP Forum. (2001, October 20). Wag uaprof. Retrieved from http://www.openmobilealliance.org/tech/affiliates/wap/wap-248-uaprof-20011020-a.pdf.

Weiser, M. (1991). The computer for the 21st century, Scientific American, 265(3). 